\section{Lateral and Chemical Force Microscopy Mapping Surface Friction and Adhesion}

M.G. Heaton, C.B. Prater, K.J. Kjoller, Digital Instruments

Lateral Force Microscopy (LFM) is a scanning probe microscopy (SPM) technique that identifies and maps relative differences in surface frictional characteristics. It is one of several techniques developed as extensions to the basic topographical mapping capabilities of SPM. LFM is particularly useful for differentiating among materials on surfaces. Applications include identifying transitions between different components in polymer blends, composites and other mixtures, identifying organic and other contaminants on surfaces, delineating coverage by coatings and other surface layers, and chemical force microscopy using functionalized tips.

\section{The Technique}

In standard contact mode atomic force microscopy (AFM), the probe is scanned over the surface (or the sample is scanned under the probe) in an $x-y$ raster pattern. A laser focused on the cantilever (the substrate which supports the probe tip) is reflected onto a segmented photodiode detector to monitor the deflection of the cantilever due to surface topography. A feedback loop maintains a constant force on the sample by adjusting the height of the cantilever to compensate for topographical features. The result is a three-dimensional map of the sample surface

With the lateral force technique, the probe is scanned perpendicular to its length; i.e., sideways on the fast axis and forward-back on the slow axis. The torsion, or twisting, of the cantilever supporting the probe will increase or decrease depending on the frictional characteristics on the surface (greater torsion results from increased friction). Since the laser detector has four quadrants (Figure 1), it can simultaneously measure and record topographic data and lateral force data. Both of these data sets can be viewed as side-by-side images in real time, as well as stored and processed independently.

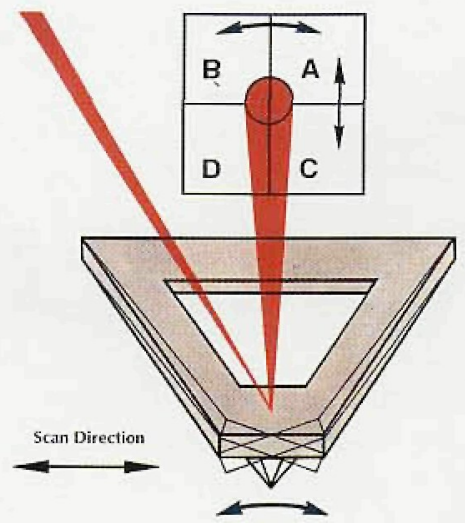

Figure 1: Scanning and detection with lateral force microscopy. For LFM, the probe is scanned sidewise and the friction signal is calculated as $(A+C)-(B+D)$. The degree of torsion of the cantilever supporting the probe is a relative measure of the surface friction caused by the lateral force exerted on the scanning probe. Note that for contact mode, the deflection signal is calculated as laser spot intensity for quadrants $(A+B)-(C+D)$.
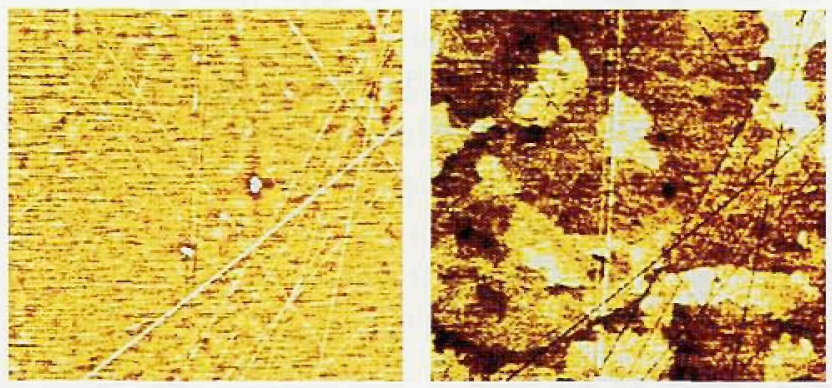

Figure 2: LFM (right) and topographical (left) images of the surface of a polished polycrystalline silicon carbide film. The polishing process obscures the grain structures in the topograhical image but it is clearly visible in the LFM image. $30 \mu \mathrm{m}$ scan

\section{Examples}

LFM can be extremely useful for identifying surface compositional differences where the materials have differing frictional characteristics and the topography is relatively undifferentiated. It should be noted, however, that these differences can be obscured by rough topography or by contamination overlying the actual sample surface.

In Figure 2, the LFM image of a polished polycrystalline silicon carbide film identifies individual grains not seen in the topographical image. Figures 3,4 and 5 show the improvement in image detail with LFM (relative to topography) for a rubber blend, a Langmuir-Blodgett film, and a magnetic recording head ceramic.

\section{Chemical Force Microscopy}

A new use of LFM is as a Chemical Force Microscope (CFM), where the tip is functionalized with one chemical species and scanned over a sample to detect adhesion differences between the species on the tip and those on the surface of the sample. Using a standard NanoScope ${ }^{(i)}$ III controller and Multimode $^{T M}$ microscope, Frisbie et al. ${ }^{1}$ changed the chemical species on the tip between scans of the same surface, causing the lateral force image of the surface to invert (Figure 6). This groundbreaking study opens an entirely new area for lateral force measurements of functional group microstructure in polymers and other materials and binding/recognition interactions in biological systems.

\section{Summary}

Lateral Force Microscopy is an SPM technique for differentiating surface compositional variations. LFM has important uses for semiconductors, polymers, deposited films, data storage devices, investigative studies of surface contamination, chemical speciation and frictional characteristics, and a growing list of new applications

Frisbie CD, Rozsnyai A, Noy A, Wrighton MS, and Lieber CM, 1994 . Functional group imaging by chemical force microscopy. Science 265: 2071-2074.
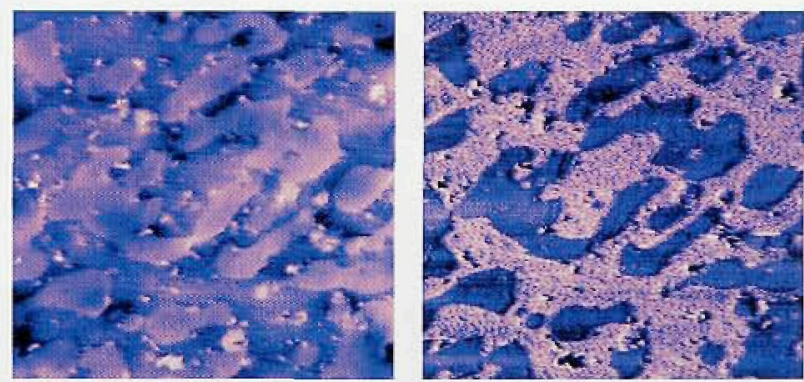

Figure 3: LFM (right) and topographical (left) images of a natural rubber/EDPM blend. 12 $\mu \mathrm{m}$ scan
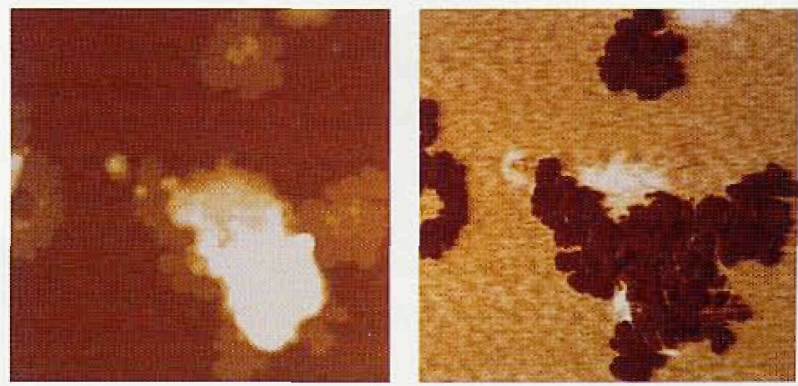

Figure 4: LFM (right) and topographical (left) images of a Langmuir-Blodgett film. $1 \mu \mathrm{m}$ scan
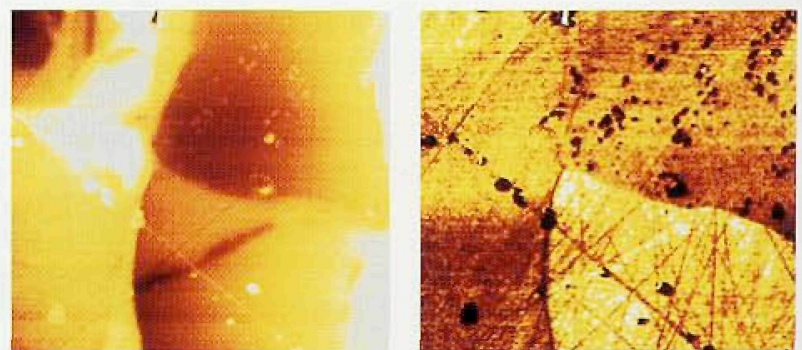

Figure 5: Air bearing surface of a magnetic recording head showing LFM (right) and topography (left) of Al oxide grains and contamination. $800 \mathrm{~nm}$ scan. 


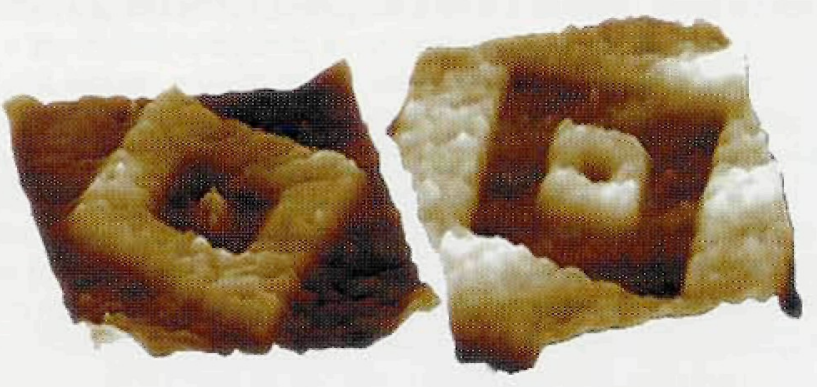

Figure 6. CFM scan of well-defined regions that terminate in either methyl or carboxylic acid groups. When a carboxylic acid-terminated tip is used for imaging (left), the carboxylic acid-terminated regions exhibit greater frictional force (lighter color) than the methylterminated regions. When a methyl-terminated tip is used (right), the friction contrast is reversed. No differences are revealed by the topographical AFM scan since the functional groups are structurally quite similar. $50 \mu \mathrm{m}$ images acquired using unmodified NanoScope MultilMade AFM system, courtesy Dr. C. Lieber, Harvard University.

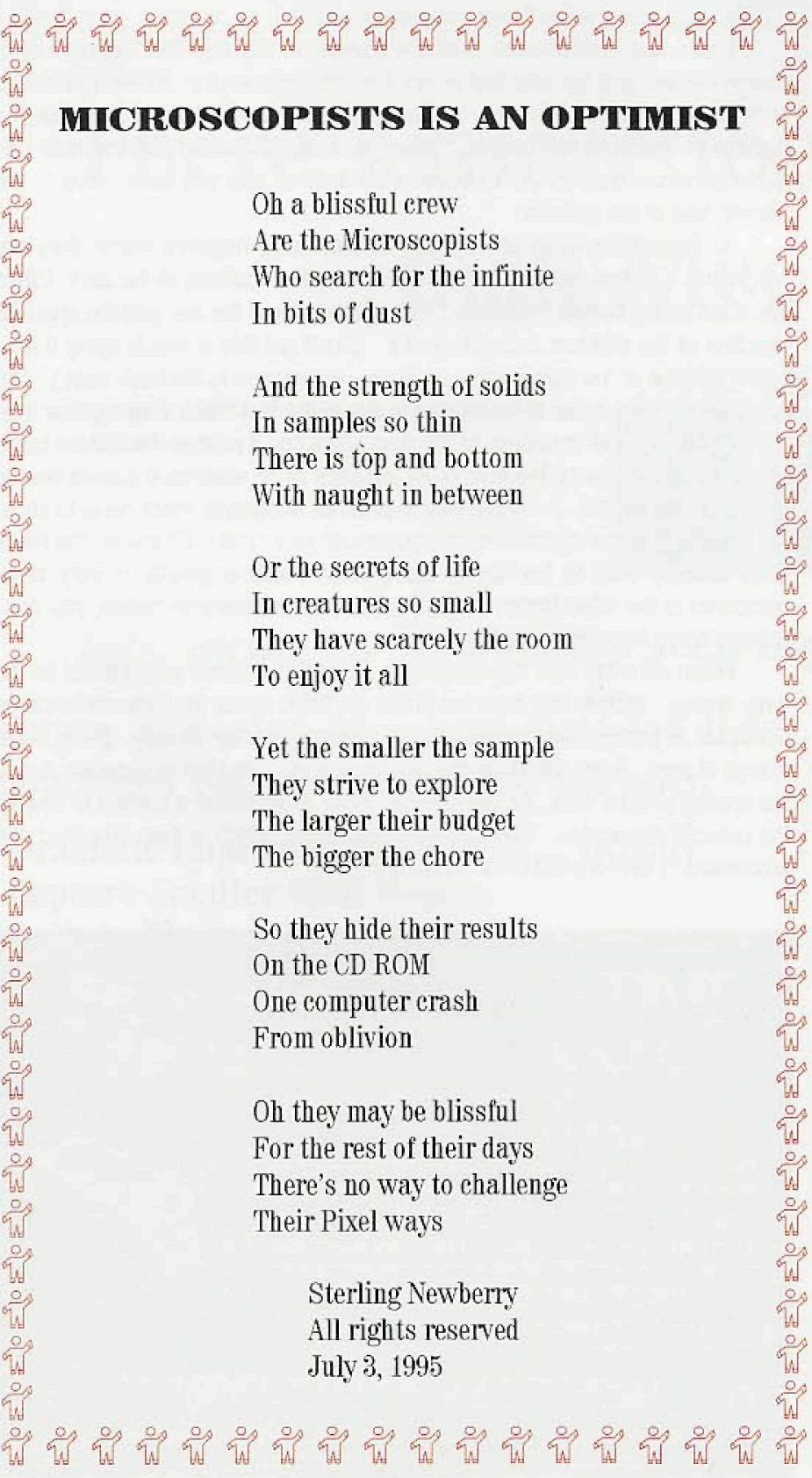

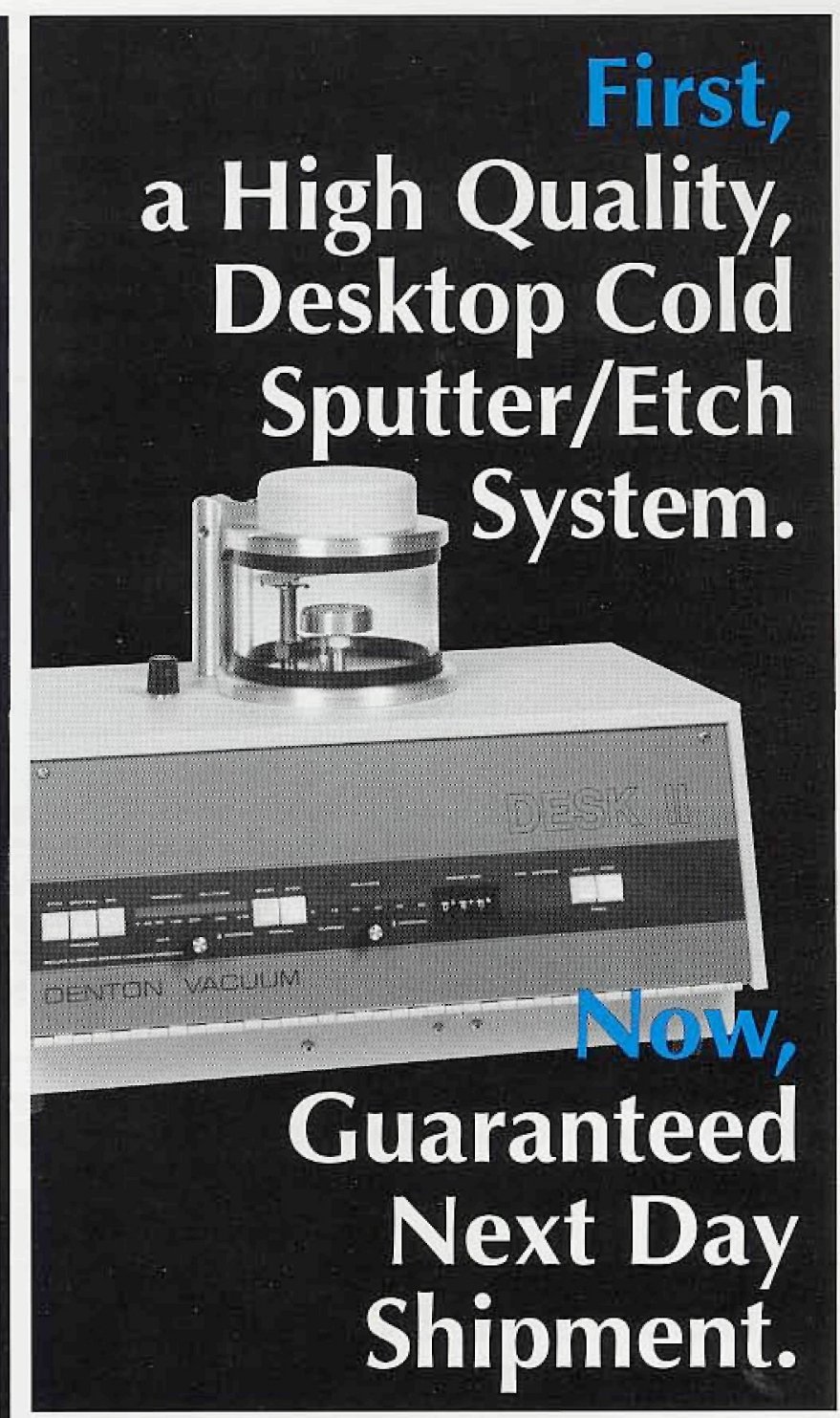

Order Denton's standard Desk II System by noon today and get guaranteed shipment tomorrow or Denton pays the freight.

The Desk II produces uniform, conductive, fine grain Au/AuPd coatings in under three minutes and is the highest quality desktop system available.

\section{The Desk // features:}

- Automatic or manual operation

- Easy to read digital vacuum and current gauges

- Optional carbon evaporation accessory

For more information, look no further than Denton... where both Quality and Delivery invite comparison.

DATON $\begin{aligned} & 1259 \text { North Church St. } \\ & \text { Moorestown, NJ 08057 USA } \\ & \text { Tel: (609) 439-9100 } \\ & \text { FAX:(609) 439-9111 }\end{aligned}$
Next day shipment must be requested at time of order.
Decades of Experience In EM Specimen Prep Equipment

